\title{
A general approach for the calculation of intermodulation distortion in cavities with superconducting endplates
}

\author{
J. Mateu, C. Collado, O. Menéndez, and J. M. O'Callaghan ${ }^{\text {a) }}$ \\ Universitat Politecnica de Catalunya, Campus Nord UPC-D3, clo Jordi Girona 1, 08034 Barcelona, Spain
}

(Received 1 July 2002; accepted 15 November 2002)

\begin{abstract}
We report on a general procedure to calculate intermodulation distortion in cavities with superconducting endplates that is applicable to the dielectric-loaded cavities currently used for measurement of surface resistance in high-temperature superconductors. The procedure would enable the use such cavities for intermodulation characterization of unpatterned superconducting films, and would remove the uncertainty of measuring intermodulation on patterned devices, in which the effect of patterning damage might influence the outcome of the measurements. We have verified the calculation method by combining superconducting and copper endplates in a rutile-loaded cavity. (C) 2003 American Institute of Physics. [DOI: 10.1063/1.1535742]
\end{abstract}

Microwave nonlinearities are important features of superconducting thin films that might limit their applicability. This is the case in high-temperature superconductors (HTS), whose use in wireless and other communication systems is limited, among other factors, by this type of nonlinearity. ${ }^{1}$ Microwave nonlinearities are enhanced when there are high current densities in the superconductor, and are most prominent in planar resonators in which magnetic field wraps around a thin superconducting film. ${ }^{2}$ There is a widespread agreement to attribute the nonlinearities to a dependence of the surface impedance on the local magnetic field or, equivalently, on the surface current density. ${ }^{3}$

There is concern regarding the possible effects of patterning superconducting films on the nonlinear behavior of the resulting planar devices, especially when considering intermodulation distortion (IMD). Besides the obvious technological implications in device fabrication, this could also affect basic studies that use the IMD of patterned resonators to probe the properties of superconductors. ${ }^{4}$ Measurements of third-harmonic generation made on planar HTS lines of various cross sections ${ }^{5}$ suggest that etching in HTS has a limited effect on IMD, but to completely rule out these effects, a study similar to Ref. 6 should be made. That work compares nonlinear measurements of cavities with superconducting films used as endplates with measurements of planar devices fabricated with the same films, and finds that the power dependence of the microwave surface resistance is unaffected by the patterning process. To extend such work to IMD and rule out the effects of patterning on this type of distortion, it is necessary to relate the results of a cavity IMD measurement with the properties of the superconducting film. In this letter, we address this by describing a method to calculate the power of the intermodulation products coupled out of a cavity with one or two superconducting endplates, and show experimental results that verify the method.

We assume that we have a cavity, which might be dielectric-loaded as those in Refs. 6 and 7, in which one of the endplates is made of a superconducting material and the

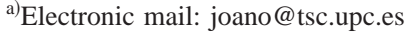

other is a normal metal. We also assume that the electric field on the superconducting endplate will be a superposition of that produced by the small-signal surface impedance of the material plus an additional component that will depend on the surface current density $\mathbf{j}_{s}$ through the equation: $\mathbf{e}_{\mathrm{NL}}$ $=\Delta R_{\alpha}\left|j_{s}\right|^{\alpha} \mathbf{j}_{s}+\Delta L_{\alpha} d / d t\left(\left|j_{s}\right|^{\alpha} \mathbf{j}_{s}\right)$ where $\Delta R_{\alpha}, \Delta L_{\alpha}$, and $\alpha$ are parameters that characterize the nonlinearities of the superconductor. This field accounts for the departure of the surface impedance from its small-signal values, which can be quantified by the parameters $\Delta R_{s}$ and $\Delta X_{s} .{ }^{8}$ These parameters can be related to the nonlinear field $\mathbf{e}_{\mathrm{NL}}$ by assuming that the surface current $j_{s}$ is sinusoidal $\left[j_{s}=J_{s} \cos \left(\omega_{0} t\right)\right]$, and finding the ratio between its amplitude and that of the fundamental of the electric field:

$$
\begin{aligned}
& \Delta R_{s}\left(J_{s}\right)=\frac{1}{J_{s}} \frac{\omega_{0}}{\pi} \Delta R_{\alpha} \int_{0}^{2 \pi / \omega_{0}}\left|j_{s}\right|^{\alpha} j_{s} \cos \omega_{0} t d t, \\
& \Delta X_{s}\left(J_{s}\right)=\frac{\omega_{0}}{J_{s}} \frac{\omega_{0}}{\pi} \Delta L_{\alpha} \int_{0}^{2 \pi / \omega_{0}}\left|j_{s}\right|^{\alpha} j_{s} \cos \omega_{0} t d t .
\end{aligned}
$$

Thus, the equation for $\mathbf{e}_{\mathrm{NL}}$ above can be made consistent with studies claiming that the reactive nonlinearities are dominant in the generation of intermodulation product ${ }^{9-11}$ by making $\Delta R_{\alpha}$ sufficiently small. Equation (2) can also account for a quadratic or linear variation of the inductance (or $\Delta X_{s}$ ) with current density $J_{s}$, as predicted for intrinsic nonlinearities in $\operatorname{HTS}^{10}(\alpha=2)$, or for nonlinearities due to other effects such as Josephson vortices ${ }^{11}(\alpha=1)$.

If the cavity is at resonance, the current density on the superconducting film will be $\mathbf{j}_{s}(\mathbf{r}, t)=A(t) f(\mathbf{r}) \hat{u}(\mathbf{r})$ where $f(\mathbf{r}) \hat{u}(\mathbf{r})$ is the spatial distribution of the resonant mode, and $A(t)$ is its time dependence, which is $A(t)=j_{1} \cos \omega_{1} t$ $+j_{2} \cos \omega_{2} t$ for the case of a two-tone intermodulation experiment. Combining the equations for $\mathbf{e}_{\mathrm{NL}}$ and $\mathbf{j}_{s}(\mathbf{r}, t)$ we find that the electric field at $2 \omega_{1}-\omega_{2}$ on the endplate is given by:

$$
\begin{aligned}
\mathbf{E}_{2 \omega_{1}-\omega_{2}}= & {\left[\Delta R_{\alpha}+j\left(2 \omega_{1}-\omega_{2}\right) \Delta L_{\alpha}\right] } \\
& \times T_{2 \omega_{1}-\omega_{2}}\left(j_{1}, j_{2}, \alpha\right) j_{1}^{\alpha} j_{2}|f(\mathbf{r})|^{\alpha} f(\mathbf{r}) \hat{u}(\mathbf{r}),
\end{aligned}
$$




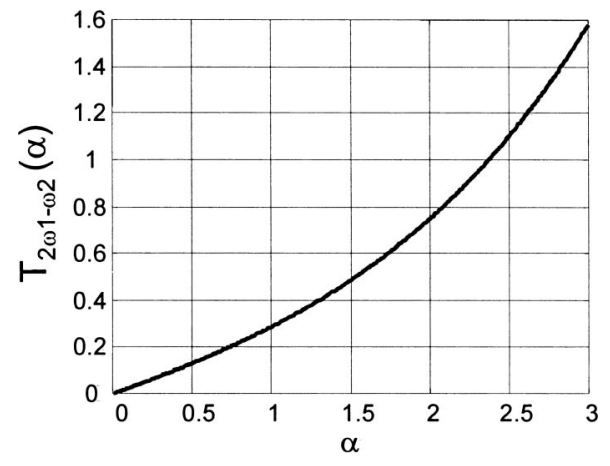

FIG. 1. Dependence of the parameter $T$ with the order of the nonlinearity $\alpha$ when the amplitude of the signals at $\omega_{1}$ and $\omega_{2}$ are kept balanced $\left(j_{1}\right.$ $\left.=j_{2}\right)$. In this situation, the amplitude of the electric field on the superconducting endplate is proportional to $T(\alpha) j_{1}^{\alpha+1}$.

where $T_{2 \omega_{1}-\omega_{2}}\left(j_{1}, j_{2}, \alpha\right) j_{1}^{\alpha} j_{2}$ is the intermodulation product at $2 \omega_{1}-\omega_{2}$ produced by the nonlinear term $|A(t)|^{\alpha} A(t)$, that is,

$$
\begin{aligned}
& T_{2 \omega_{1}-\omega_{2}}\left(j_{1}, j_{2}, \alpha\right) j_{1}^{\alpha} j_{2} \\
& \quad=\lim _{T \rightarrow \infty} \frac{2}{T} \int_{-T / 2}^{T / 2}|A(t)|^{\alpha} A(t) e^{-j\left(2 \omega_{1}-\omega_{2}\right) t} d t .
\end{aligned}
$$

Note that, as shown in Fig. 1, $T_{2 \omega_{1}-\omega_{2}}\left(j_{1}, j_{2}, \alpha\right)$ only depends on $\alpha$ when the amplitudes of the two signals at $\omega_{1}$ and $\omega_{2}$ are kept balanced $\left(j_{1}=j_{2}\right)$. Note also that Eq. (3) can be written as $\mathbf{E}_{2 \omega_{1}-\omega_{2}}=E_{2 \omega_{1}-\omega_{2}}|f(\mathbf{r})|^{\alpha} f(\mathbf{r}) \hat{u}(\mathbf{r})$; that is, $|f(\mathbf{r})|^{\alpha} f(\mathbf{r})$ is the spatial dependence of the electric field on the endplate. This field will excite several modes in the cavity, among them the mode at which $\omega_{1}$ and $\omega_{2}$ resonate. This particular mode has a magnetic field on the surface of the superconductor whose spatial distribution is $f(\mathbf{r})$; that is, $\mathbf{H}_{2 \omega_{1}-\omega_{2}}=H_{2 \omega_{1}-\omega_{2}} f(\mathbf{r}) \hat{v}(\mathbf{r})$, where $\hat{v}(\mathbf{r})$ is a unit vector parallel to the surface and perpendicular to $\hat{u}(\mathbf{r})$. To determine $H_{2 \omega_{1}-\omega_{2}}$, we balance the power generated at the surface at $2 \omega_{1}-\omega_{2}$ with the power that is dissipated in the cavity or coupled out of it; that is,

$$
P=\frac{1}{2} \int_{S} \mathbf{E}_{2 \omega_{1}-\omega_{2}} \times \mathbf{H}_{2 \omega_{1}-\omega_{2}}^{*} d \mathbf{S}=\frac{\left(2 \omega_{1}-\omega_{2}\right) W}{Q_{L}},
$$

where the integral is done across the surface of the superconductor, $W$ is the energy stored in the resonator, and $Q_{L}$ is its loaded $Q$. Equation (5) can be transformed to

$$
\begin{gathered}
\frac{1}{2} E_{2 \omega_{1}-\omega_{2}} H_{2 \omega_{1}-\omega_{2}}^{*} \int_{S}|f(\mathbf{r})|^{\alpha} f(\mathbf{r}) f(\mathbf{r}) d S \\
=\frac{\left(2 \omega_{1}-\omega_{2}\right) W_{0}}{Q_{L}} H_{2 \omega_{1}-\omega_{2}}^{*} H_{2 \omega_{1}-\omega_{2}},
\end{gathered}
$$

where $W_{0}=W /\left|H_{2 \omega_{1}-\omega_{2}}\right|^{2}$. The value of $H_{2 \omega_{1}-\omega_{2}}$ follows from Eq. (6):

$$
H_{2 \omega_{1}-\omega_{2}}=\frac{1}{2} E_{2 \omega_{1}-\omega_{2}} \frac{Q_{L}}{\left(2 \omega_{1}-\omega_{2}\right) W_{0}} \int_{S}|f(\mathbf{r})|^{\alpha} f(\mathbf{r}) f(\mathbf{r}) d S,
$$

where where
Downloaded 24 Jun 2007 to 147.83.95.32. Redistribution subject to AIP license or copyright, see http://apl.aip.org/apl/copyright.jsp

$$
\begin{aligned}
E_{2 \omega_{1}-\omega_{2}}= & {\left[\Delta R_{\alpha}+j\left(2 \omega_{1}\right.\right.} \\
& \left.\left.-\omega_{2}\right) \Delta L_{\alpha}\right] T_{2 \omega_{1}-\omega_{2}}\left(j_{1}, j_{2}, \alpha\right) j_{1}^{\alpha} j_{2} .
\end{aligned}
$$

Equations (4), (7), and (8) contain all the necessary information to calculate the power of the intermodulation product coupled out of the cavity from the available power of the sources producing the signals at $\omega_{1}$ and $\omega_{2}$. All that is needed are the nonlinear parameters of the films $\left(\Delta R_{\alpha}, \Delta L_{\alpha}, \alpha\right)$, the field distribution in the cavity $\left[W_{0}\right.$ and $f(\mathbf{r})]$, its quality factor $Q_{L}$, the proportionality factor between the power available at $\omega_{1}$ and $j_{1}^{2}$ (which is the same as that relating the power available at $\omega_{2}$ and $j_{2}^{2}$ ), and the proportionality factor relating $\left|H_{2 \omega_{1}-\omega_{2}}\right|^{2}$ and the power delivered to the instrumentation at $2 \omega_{1}-\omega_{2}$. Except for $\Delta R_{\alpha}$, $\Delta L_{\alpha}$, and $\alpha$, all these can be calculated using standard techniques for cavity analysis.

Note that, since $T_{2 \omega_{1}-\omega_{2}}\left(j_{1}, j_{2}, \alpha\right)$ only depends on $\alpha$ when $j_{1}=j_{2}$, Eqs. (7) and (8) imply that the power of the intermodulation products will grow with a slope $\alpha+1$ with respect to the power of the sources at $\omega_{1}$ and $\omega_{2}$. Thus, our analysis is consistent with studies on HTS nonlinearities, ${ }^{10,11}$ which claim that this slope is 3:1 when there is a square-law dependence of the inductance with the current density ( $\alpha$ $=2)$, and 2:1 when this dependence is linear $(\alpha=1)$.

This calculation is easily extended to the case in which two superconducting endplates are used if the mode is such that the current distribution on both endplates is identical (as is usually the case in HTS measurements ${ }^{6,7}$ ). The integral in Eq. (5) has to be carried over the two endplates, and $\mathbf{E}_{2 \omega_{1}-\omega_{2}}$ will be different in each integrand, reflecting the nonlinearities of the two films being tested. Therefore, the total value of $H_{2 \omega_{1}-\omega_{2}}$ can be obtained by applying twice the formulas for a single superconducting endplate and summing the resulting values of $H_{2 \omega_{1}-\omega_{2}}$.

To check the procedure, we have measured two state-ofthe-art $\mathrm{Y}-\mathrm{Ba}_{2}-\mathrm{Cu}_{3}-\mathrm{O}_{7-\delta}(\mathrm{YBCO})$ samples produced by a commercial supplier (Theva). The YBCO films, 700-nm thick, were deposited onto $10 \times 10 \mathrm{~mm}^{2} \mathrm{MgO}$ substrates with a thickness of $0.5 \mathrm{~mm}$, and were used as delivered, with no further processing on our part. The resonator used was similar to the one described in Ref. 7, with a rutile cylinder $4 \mathrm{~mm}$ in diameter and $3 \mathrm{~mm}$ in height. We made one-port IMD power measurements using copper in one of the cavity endplates and a YBCO film in the other. These measurements were done with a setup in which a spectrum analyzer is used to measure the signals going out of the resonator and in which, in the case of critical coupling, there is high isolation between the spectrum analyzer and the sources producing the signals at $\omega_{1}$ and $\omega_{2}$. Testing was done in direct liquid nitrogen immersion to avoid thermal effects in the IMD. We could not measure any change in $Q$ or resonant frequency with source power. This is consistent with the maximum values of $\Delta R_{s}$ and $\Delta X_{s}$ that result from Eqs. (1) and (2) when IMD power data is fitted to our model and either dominant resistive nonlinearities $\left[\Delta R_{\alpha} \gg \omega \Delta L_{\alpha}\right.$, see Eq. (3)] or dominant reactive nonlinearities $\left(\Delta R_{\alpha} \ll \omega \Delta L_{\alpha}\right)$ are assumed. We will hereafter assume that reactive nonlinearities are dominant ${ }^{9-11}$ but, by Eq. (3), the results are valid for any pair 


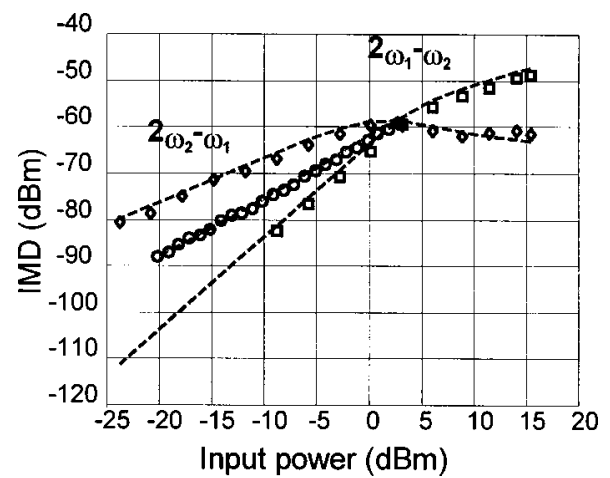

FIG. 2. Results of measurements and calculations for a rutile resonator with two superconducting endplates where $Q_{0}=80000$ and $Q_{L}=17000$. Circles represent the measured power of the intermodulation product at $2 \omega_{1}-\omega_{2}$ as a function of the source power when the power of both sources was increased simultaneously. Squares and diamonds represent the measured power of the intermodulation products at $2 \omega_{1}-\omega_{2}$ and $2 \omega_{2}-\omega_{1}$ respectively, as a function of the source power at $\omega_{1}$ when the power of the source at $\omega_{2}$ was kept constant $(3 \mathrm{dBm})$. The lines in the figure are results of calculations performed with parameters obtained from measurements were only a single HTS film was used in the resonator, and the other endplate was a normal metal (OHFC copper).

this assumption we obtain $\alpha=1$ and $\Delta L_{\alpha}=3.5$ $\times 10^{-19} \mathrm{Hm} / \mathrm{A}$ for one of the films and $\alpha=0.2$ and $\Delta L_{\alpha}=5 \times 10^{-16} \mathrm{Hm}^{0.2} \mathrm{~A}^{0.2}$ for the other. The parameters of the first film are comparable with those of Ref. 12, which are also intermodulation measurements on unpatterned YBCO, from which values of $\alpha=1$ and $\Delta L_{\alpha}=1.2 \times 10^{-19} \mathrm{HmA}^{-1}$ can be inferred. ${ }^{13}$

To further verify the model, we also made IMD measurements with both films in the resonator, and compared them with the calculations made using $\Delta L_{\alpha}$ and $\alpha$ extracted from the previous measurements. Figure 2 shows the results in which measurements (points) are compared to calculations (lines), showing that the IMD of the resonator with two HTS films can be predicted from that of the two resonators having one HTS film and a copper endplate (i.e., there is no direct data fitting done in Fig. 2).

In conclusion, we have shown how to relate the results of an intermodulation measurement in a cavity with the nonlinear parameters of a superconducting film (not necessarily
HTS) for the case in which the nonlinearity can be assumed to arise from a stray electric field at the surface whose dependence on current density follows the equation $\mathbf{e}_{\mathrm{NL}}$ $=\Delta R_{\alpha}\left|j_{s}\right|^{\alpha} \mathbf{j}_{s}+\Delta L_{\alpha} d / d t\left(\left|j_{s}\right|^{\alpha} \mathbf{j}_{s}\right)$. A similar technique can be used to extend this work for other types of nonlinearity, or to consider the case where both the resistive and reactive parts of the nonlinearity play a role in the IMD, but they follow different dependences on $j_{s}$. The experimental verification of the method reveals differences in IMD performance between two nominally identical films supplied by the same vendor, which proves the need to use methods of nondestructive IMD screening of the films like the one described in this work.

This work has been funded by Spanish Ministry of Education and Culture through project no. MAT99-0984-C03-03 and scholarship AP99 78085980, and by the Generalitat de Catalunya through scholarship 2002 FI 00622.

${ }^{1} \mathrm{~S}$. H. Talisa, in Microwave Superconductivity edited by H. Weinstock, M. Nisenoff (Kluwer Academic, Dordrecht, 2001), Chap. 8, pp. 189-204; B. A. Willemsen, ibid., Chap. 15, pp. 387-416.

${ }^{2}$ Z.-Y. Shen, C. Wilker, P. Pang, D. W. Face, C. F. Carter, and C. M. Harrington, IEEE Trans. Appl. Supercond. 7, 2446 (1997).

${ }^{3}$ D. E. Oates, in Microwave Superconductivity edited by H. Weinstock, M. Nisenoff (Kluwer Academic, Dordrecht, 2001), Chap. 5, pp. 117-131; M. A. Hein, ibid., Chap. 2, pp. 34-45.

${ }^{4}$ G. Benz, S. Wünsch, T. A. Scherer, M. Neuhaus, and W. Jutzi, Physica C 356, 122 (2001).

${ }^{5}$ J. C. Booth, J. A. Beall, D. A. Rudman, L. R. Vale, and R. H. Ono, J. Appl. Phys. 86, 1020 (1999).

${ }^{6}$ X. Hao, D. E. Oates, A. C. Anderson, A. R. L. Slattery, G. Dresselhaus, and M. S. Dresselhaus, IEEE Trans. Microwave Theory Tech. 48, 1221 (2000).

${ }^{7}$ N. Klein, C. Zuccaro, U. Dähne, H. Schulz, N. Tellmann, R. Kutzer, A. G. Zaitsev, and R. Wördenweber, J. Appl. Phys. 78, 6683 (1995).

${ }^{8}$ J. S. Herd, D. E. Oates, and J. Halbritter, IEEE Trans. Appl. Supercond. 7, 1299 (1997).

${ }^{9}$ T. Dahm, D. J. Scalapino, and B. A. Willemsen, J. Appl. Phys. 86, 4055 (1999).

${ }^{10}$ T. Dahm and D. J. Scalapino, J. Appl. Phys. 81, 2002 (1997).

${ }^{11}$ T. Dahm and J. Oppenländer, IEEE Trans. Appl. Supercond. 11, 1392 (2001).

${ }^{12}$ S. Kolesov, H. Chaloupka, A. Baumfalk, and T. Kaiser, J. Supercond. 10, 179 (1997).

${ }^{13}$ C. Collado, J. Mateu, T. J. Shaw, and J. M. O'Callaghan, Physica C 372376, 566 (2002). 